\title{
A framework to evaluate the educational potential of a digital artefact for math learning
}

\author{
Giampaolo Chiappini
}

Istituto per le Tecnologie Didattiche (ITD), CNR, Italy, chiappini@itd.cnr.it,0000-0003-4847-972X

\begin{abstract}
This article provides a methodology with two potential applications: to prove useful to maths teachers for analysing and evaluating the educational potential of different digital artefacts and to help designers of maths learning artefacts to evaluate their design during the implementation phase. The educational potential of an artefact is considered as an entity determined by actions and representations structure available within the artefact, the interpretation and behaviour of who uses it and the features of the activity in which it is used. The proposed methodology is based on the notions of affordance, narrative and cycle of expansive learning. The methodology has been applied on AlNuSet, a system designed for supporting the teaching and learning of algebra by means of modalities of interaction that are of visual, spatial and motor nature.
\end{abstract}

Keywords: Mathematics, Computer-assisted learning

\section{Introduction}

The problem of how to evaluate the effectiveness of a digital artefact is addressed in the context of human-computer interaction (HCI) research through the notion of usability and its evaluation (Norman, 1988; 1993; Norman \& Draper, 1986; Whitefield, Wilson, Dowell, 1991; McGrenere \& Ho, 2000; Nielsen, 1995). According to the definition given in ISO 9241 part 11, usability is 'the extent to which a product can be used by specified users to achieve specified goals with effectiveness, efficiency, and satisfaction in a specified context of use.' We observe that the methods so far developed in the field of HCI for assessing usability are unsuitable in cases where the user does not have sufficient grasp of task objectives and the domain or if he pursues goals that go beyond those inherent in the task.

The context of use at the centre of our attention is the teaching and learning of mathematics within the constraints of the school system. Here, both students and teacher are to be considered as users; the objectives they pursue via artefact use are distinct but tightly interwoven. Students generally use the artefact to perform a mathematical task assigned to them by the teacher. They might not have a clear idea of the objectives of the task to be performed, and above all, they cannot be considered as experts of the domain which the task addresses (Bottino \& Chiappini, 1998). The teacher uses the artefact as a means to create the conditions by which students can develop specific skills and can appropriate theoretical and practical knowledge. These often transcend the strict confines of the specific task at hand, which represents only a single facet of the broader context and agenda, namely, the general mathematical formation of the student. In this multi-tiered context of use of the artefact the standard HCI methods for evaluating artefact usability are unfit to evaluate the potentiality of the artefact to promote student's progress toward educational goals that do not coincide necessarily with the goals of the task but that could become actual by virtue of the artefact-mediated activity (Bottino \& Chiappini, 1998).

In this work, this potentiality of the artefact is defined as Educational Potential of the Artefact.

The author considers the educational potential of an artefact for the learning of mathematics as an entity that is determined equally by (i) the structure of actions and representations available within the artefact (embedded by the designer), (ii) the interpretation and behaviour of who uses it and (iii) the features of the activity in which it is used.

This work is aimed to present the methodology that has been elaborated to evaluate the educational potential of an artefact in the field of mathematics by considering the aspects (i), (ii) and (iii) reported earlier as being tightly interwoven. This methodology is based on the concept of affordance developed in the context of HCI, integrated with the notion of narrative developed by Bruner $(1991,1997)$ and with the notion of cycle of expansive learning developed by Engeström (1987) and Engeström \& Sannino (2010). This study has been accomplished with reference to a specific 


\section{A framework to evaluate the educational potential of a digital artefact for math learning \\ Chiappini}

software, the system AlNuSet, developed within the ReMath European project (Lagrange \& Kynigos, 2014; Lagrange $\&$ Chiappini, 2007) to support the process of algebra teaching and learning.

\section{Affordance as a conceptual tool for describing the artefact's action and representation structure}

Gibson (1979) introduced the concept of affordance in perceptual psychology studies to denote the relation between the organism and its environment. Gibson's affordances are very basic kinds of relationships, such as physical surfaces that provide support or objects that can be manipulated, grasped, cut, scraped, etc. Gibson stated that an affordance is equally a part of the environment and behaviour and it may be detected and used without explicit awareness of doing so, as in the case of a pavement.

Subsequently, the concept of affordance was explored by Norman (1988) and generally adopted in the field of HCI. Here it " refers to the perceived and actual properties of the thing, primarily the fundamental properties that determine just how the thing could possibly to be used" (Norman, 1988).

The examples of affordance considered in this field of research are more complex than those considered by Gibson. For this reason, Turner (2005) distinguished between simple affordances (according to Gibson's view) and complex affordances (such as those of a digital artefact). He stated that the latter embody things such as history and practice and hence encapsulate meanings, values and principles of the cultural domain in which they are used.

For the evaluation of simple and complex affordances, Turner and Turner (2002) have elaborated a framework centred on a three-layer articulation of their complexity and have distinguished the affordances amongst perceived affordances, ergonomic affordances, cultural affordances. In this work, we use this framework to evaluate the affordances of the Algebraic Line (AL) of AlNuSet.

AlNuSet is a system that has been designed to enhance the processes of teaching and learning of algebra (Chiappini, 2014; Pedemonte \& Chiappini, 2008). AlNuSet is constituted by three environments strictly integrated amongst them: the AL, the Algebraic Manipulator, and the Functions environment. In this work, only the AL is considered.

The $\mathrm{AL}$ is a standard number line that has been enhanced with new complex affordances for:

- Building algebraic expressions on the line, using geometric models of operations;

- Exploring properties of algebraic expressions on the AL;

- Searching for the roots of polynomials with integer coefficients;

- Exploring and defining the truth sets of algebraic propositions.

According to the Turner and Turner framework, the AL makes available several perceived affordances that are fully controllable in visuospatial and motor terms by means of buttons, sliders, physical affordances of the mouse to move between the environments, graphical models and an edit field. Perceived affordances concern the characteristics of the controls that mediate interaction with the system (Turner \& Turner, 2002).

To give you an idea of the perceived affordances of the AL of AlNuSet, consider its interface that is structured by two identical straight lines with positive and negative integers associated with points that are equidistant from each other as shown in Fig 1a.

These lines are called Algebraic Lines because it is possible to insert on them a point associated to a letter (for example, the letter $\mathrm{x}$ ) by means of an edit field as in the example reported in Fig $1 \mathrm{~b}$.

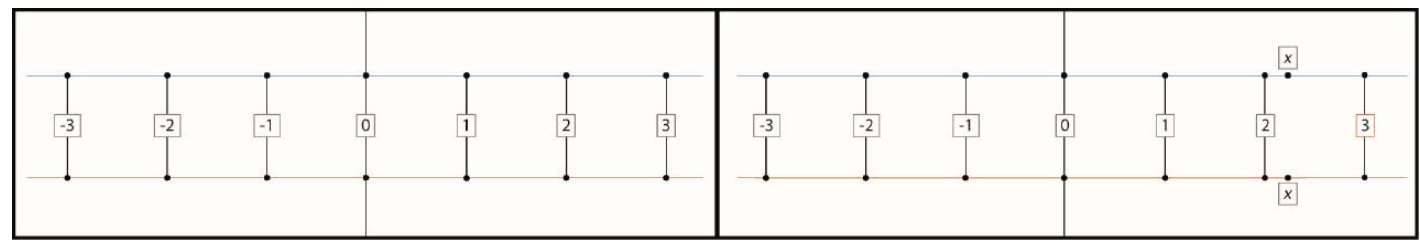

(a)

(b)

Fig. 1 Example of a perceived affordance on the AL.

Through mouse-driven exploration and interaction, the user can perceive that it is possible to drag the point associated to the letter $\mathrm{x}$ left or right along the line. As it will be shown further in the following text, the drag of a variable point is a perceived affordance that plays an important role in terms of education. The interaction with this perceived affordance requires only that the user mobiles his/her spatial, visual and motor experience. At this level, evaluation of perceived affordance concerns the assessment of usability (in the sense of Norman) of the basic elements that the user perceives as being available to control interaction with the system.

In the Turner and Turner framework, the middle level is constituted by the ergonomic affordance. This concerns the embodied actions that the user can detect within, and pursue through, the artefact when tackling tasks and subtasks that are specific to the context where the system is used (Turner \& Turner, 2002). To illustrate an example of ergonomic affordance related to the $\mathrm{AL}$, consider the task of verifying whether the statement ' $-\mathrm{x}$ is always a negative number' is 
correct (Chiappini, 2011). In a experimentation of AlNuSet, several students believed that the assertion was correct 'because $\mathrm{x}$ has a minus sign in front of it'. The teacher then got the students to verify their hypothesis using the AL.

To solve the task, these students, first, represent $\mathrm{x}$ and $-\mathrm{x}$ on the algebraic line and then they drag $\mathrm{x}$ through increasing positive values, verifying that for these values, $-x$ assumes negative values on the line and then through decreasing positive values, and finally through negative values. The use of the drag allowed the students to experience that if $x$ is negative, $-x$ denotes positive values, which comes as an effective surprise. In this way, they realised that the teacher's original statement was incorrect.

The students recognised a usefulness (McGrenere \& Ho, 2000) to the narrative of the behaviour of $-\mathrm{x}$ that emerged when dragging $\mathrm{x}$ on the AL. The dragging of $\mathrm{x}$ and the corresponding narrative of the behaviour of $-\mathrm{x}$ on the AL that appeared as a retroaction of the system constitute the ergonomic affordance used by the student for the solution of the task.

The evaluation of ergonomic affordance concerns the assessment of the usefulness (McGrenere \& Ho, 2000) of the embodiment of action possibilities for task objectives that users can detect and pursue.

The third level of the Turner and Turner framework is the cultural affordance. This concerns a feature or set of features that appears from using the artefact, and in doing so, endowing it with the values of culture from which it arises, in our case the culture of algebra (Turner \& Turner, 2002).

The example of Fig. 2 shows that in the AL environment, the user can insert a letter such as $\mathrm{x}$, which is then associated with a point on the line, an expression such as $x+3$, which is then associated to a point on the line, a proposition such as $x+3=0$, which is represented in a window and associated to a coloured little circle.

If the user drags the point associated to $\mathrm{x}$ along the line, he/she can perceive that $\mathrm{x}+3$ move accordingly on the line. At the same time, he/she can perceive that when the point associated with $\mathrm{x}$ is dragged along the line, the coloured circle associated to $x+3=0$ may change colour (Fig. 2): green (white in the figure) if $x$ is placed on the point -3 and the red (black in the figure) in all other cases.

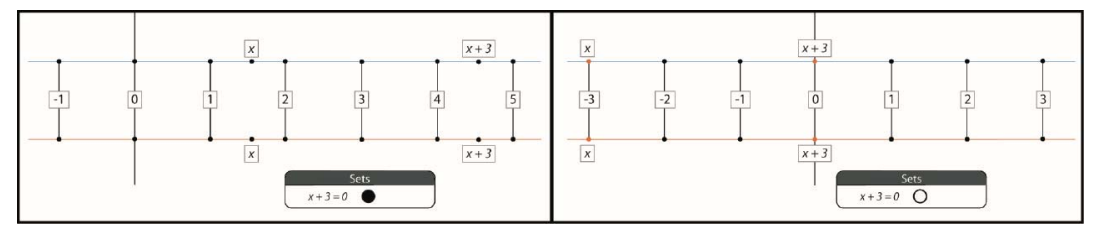

(a)

(b)

Fig. 2 Features that appears from using the AL.

In Table 1, a set of characteristics of the narratives that emerge in the interaction with the AL environment are put in correspondence with their algebraic meaning

Table 1. Correspondence between characteristics of the narrative and meaning.

\begin{tabular}{ll}
\hline \multicolumn{1}{c}{ Characteristics of the narrative } & \multicolumn{1}{c}{ Meanings } \\
\hline $\begin{array}{l}\text { The point that is associated with a letter (e.g. } \mathrm{x}) \text { is draggable with the mouse on } \\
\text { the algebraic line }\end{array}$ & Independent variable \\
The movement of an algebraic expression containing a variable (e.g. $\mathrm{x}+3)$ & Dependent variable \\
depends on the movement of the variable & Difference between what algebraic \\
The equality $\mathrm{x}+3=0$, differently from the expression $\mathrm{x}+3$, is represented in a & $\begin{array}{l}\text { denote } \\
\text { window and not on the straight line }\end{array}$ \\
The small circle associated with $\mathrm{x}+3=0$ turns green when $\mathrm{x}$ is dragged on -3 & Truth value of an algebraic proposition
\end{tabular}

The meanings represented in the second column are specific of the culture where the artefact comes from and are reflected in specific characteristics of the narratives that, in the example, emerge through the use of the drag affordance. These characteristics are cultural affordances. They are evident to experts of the algebra domain. Students have to learn to recognise them as cultural affordance.

The evaluation of the effectiveness of the cultural affordance concerns the assessment of expressiveness of the characteristic of the narrative that emerge in the use of the artefact for the construction of meanings, concepts, values and principles of the algebraic domain. 


\section{A framework to evaluate the educational potential of a digital artefact for math learning \\ Chiappini}

\section{Bruner's narrative as a complement to operationalise the evaluation of the affordances of an artefact}

In order to develop a methodology to assess the effectiveness of the affordances of AlNuSet, a method to evaluate its ergonomic and cultural affordances, but not its perceived affordance, has been researched. ${ }^{1}$

To operationalise the assessment of the usefulness and the expressiveness of the narrative emerging in the use of the artefact for the task solution (ergonomic affordance) and the construction of meanings and concepts of the discipline (cultural affordance), an instrument is necessary to analyse the narratives that can emerge in the interaction with the environment. To this aim, the universal principles of narrative construction of reality developed by Bruner (1991; 1997) can be appropriate.

Bruner (1997) pointed out that the narrative is the first device used by human beings to produce knowledge. He developed a synthesis of the main properties of narratives, which he called the universal principles of narrative construction of realities (Bruner, 1991).

A brief presentation of these universal principles are reported in Table 2.

Table 2. The universal principles of narrative construction of realities elaborated by Bruner.

\begin{tabular}{|c|c|c|}
\hline P1 & Narrative diachronicity & $\begin{array}{l}\text { A narrative is an account of events occurring over time [...] Even nonverbal media have conventions } \\
\text { of narrative diachronicity. }\end{array}$ \\
\hline P2 & Particularity & Narratives depict particular events but the particulars of narratives are tokens of broader types \\
\hline P3 & $\begin{array}{l}\text { Intentional state } \\
\text { entailment }\end{array}$ & The events that occur in the narrative must be relevant to the intentional states of the characters. \\
\hline P4 & $\begin{array}{l}\text { Hermeneutic } \\
\text { composability }\end{array}$ & $\begin{array}{l}\text { It involves the interpretation of narratives in terms of part-to-whole comparison, viewing the meaning } \\
\text { of the text as a whole as a composition of its individual components and their intended meanings. }\end{array}$ \\
\hline P5 & Canonicity and breach & $\begin{array}{l}\text { Narratives involve variations, or breaches, from normal occurrences or canonical scripts. Canonicity } \\
\text { refers to usual happenings. Breaches are the deviations that make the story worth telling. }\end{array}$ \\
\hline P6 & Referentiality & $\begin{array}{l}\text { The principle that a story in some way references reality, although not in a direct way; narrative truth } \\
\text { can offer verisimilitude but not verifiability. }\end{array}$ \\
\hline P7 & Genericness & $\begin{array}{l}\text { The flip side to particularity, this is the characteristic of narrative whereby the story can be classified } \\
\text { as a genre. }\end{array}$ \\
\hline P8 & Normativeness & $\begin{array}{l}\text { The observation that narrative in some way supposes a claim about how one ought to act. This } \\
\text { follows from canonicity and breach. }\end{array}$ \\
\hline P9 & $\begin{array}{l}\text { Context sensitivity and } \\
\text { negotiability }\end{array}$ & $\begin{array}{l}\text { The lens through which people interpret the text. Negotiation of meaning between the author and the } \\
\text { reader. }\end{array}$ \\
\hline P10 & Narrative accrual & $\begin{array}{l}\text { The manner by which stories come together to create a coherent whole. New stories come from old } \\
\text { stories. The development of narratives. }\end{array}$ \\
\hline
\end{tabular}

Bruner (1997) stated that it is possible to investigate the narrative thinking by analysing the narrative discourse through which thinking comes to life by means of the principles reported earlier.

The narratives at the centre of our interest concern the function and the behaviour of algebraic objects, such as variables, expressions and propositions of algebraic nature, which may emerge in the interaction with the AL of AlNuSet during the development of an algebraic activity.

This type of narratives can be defined as Narratives Embedded in the Use of the Artefact (NEUA). They emerge during the solution of a mathematical problem as a result of 'collaboration' between the user/s of the artefact and the artefact's designer, who have designed the artefact with the features that the artefact reveals in the use. It is important to note that the NEUA encapsulates meanings, values and principles of the cultural domain (mathematics) from which the artefact emerged; these are clearly recognisable to any expert of the domain.

Bruner's universal principles of narrative can be an effective tool to evaluate the usefulness and expressiveness of NEUA in providing an idea of algebraic objects involved in the solution of a task that is able to affect the emergence of objectives for the task to be solved and the construction of meanings, concepts, principles and values of algebra.

\footnotetext{
${ }^{1}$ To evaluate the perceived affordance, methods and guideline developed in the context of HCI for the assessment of the usability are appropriate.
} 


\section{A framework to evaluate the educational potential of a digital artefact for math learning \\ Chiappini}

In order to clarify this, in the following, some examples concerning the use of variables and algebraic expressions on the AL will be furnished.

In the $\mathrm{AL}$, the dragging of the point that is associated to a letter incorporates an interesting narrative of the algebraic variable, which is characterised by a temporal dimension (P1) because it is linked to the subject's action of moving the variable point over time. Furthermore, the dragging action assumes characteristics of canonicity (P5) that depend on the numeric set in which the AL has been instantiated. When $\mathrm{x}$ is dragged along the AL, a narrative that is both general and particular (P2) emerges: student can experience a reality whereby the letter $\mathrm{x}$ represents (in a general way) all the numbers that the point associated to $\mathrm{x}$ indicates in a deictic way whilst dragged along the line (Chiappini, Reggiani \& Angelini, 2014).

However, it is only when expressions containing variables are inserted on the AL that this environment reveals its educational potential.

Figure 3 shows two screenshots of the AL where two algebraic expressions are represented.

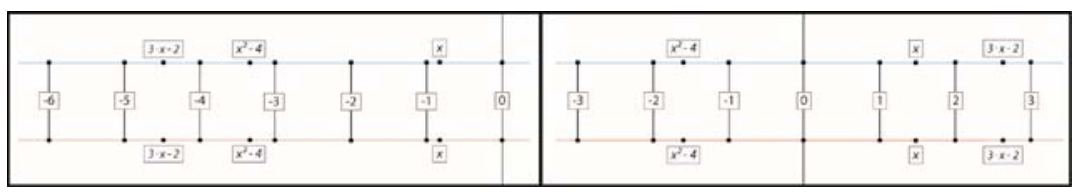

Fig. 3 Exploration of the behaviour of two algebraic expressions on the AL

It is important to note that each of the expressions is associated to a point on the line. Dragging the variable displaces the points associated to the expressions on the line. The narrative is dynamic (it has a temporal dimension linked to the action of moving the variable - P1) and renders the perceptible characteristics of 'normal processuality' (i.e. the canonical dimension of the narrative: changing the variable's value changes the value of the expression - P5). Moreover, when the variable is dragged, the expression labels move accordingly along the AL, representing all the numerical values that the associated point indicates.

This narrative, similar to that of the variable, is quantitative in nature and at the same time particular and general (P2). Finally, the dragging affordance renders a perceptible narrative that may prove useful for conceptualising the relationships established between expressions. In the Table 3, the left-hand column lists some relationships between expressions, whilst the right-hand column shows the narratives in which those relationships are expressed when the variable is dragged along the AL (Chiappini, Reggiani \& Angelini, 2014).

Table 3. Narratives of the properties of algebraic expressions on the AL.

\begin{tabular}{l}
\multicolumn{1}{c}{$\begin{array}{c}\text { Relations between } \\
\text { expressions }\end{array}$} \\
\hline $\begin{array}{l}\text { Equivalent expressions (i.e. } \\
2 x+3 x \text { and } 5 x)\end{array}$ \\
Equivalent expressions \\
excluding a finite number of \\
points (i.e. $\left(x^{2}+x\right) / x$ and \\
$x+1)$
\end{tabular}

Equivalent expressions only in a finite number of points (i.e. $2 x+3$ and $5 x$ )

Opposite expressions (i.e. $2 x$ --1 and $-2 x+1$ )

Expression that is the sum of opposite expressions (i.e. $\left.x(x-1)-x^{2}+x\right)$

Reciprocal expressions (i.e. $x /(3 x-1)$ and $(3 x-1) / x$

Expression that is product of reciprocal expressions

Expressions $E_{1}(x)$ and $E_{2}(x)$ such that $E_{1}(x)>E_{2}(x)$ for all values of $x$
Description of the narratives emerging on the $\mathrm{AL}$

Equivalent expressions are always bound to the same point on the line when dragging variables on which both expressions depend (e.g. $2 x+3 x$ and $5 x$ move associated with the same point when $x$ is dragged on the line)

These expressions coincide for each value of the variable during the drag along the line, excluding one or more values for which one of the two expressions 'disappears' from the line (e.g. the expression $\left(x^{2}+x\right) / x$ and the expression $x+1$ coincide for each value of $x$, except for the value $x=0$, for which the first expression disappears from the line, whilst the other does not).

The expressions are associated to the same point on the line only for certain values that the variable assumes during dragging on the line (e.g. the expressions $2 x+3$ and the expression $5 x$ coincide at the same point corresponding to 5 only if $x$ is dragged out of 1 )

Whilst dragging the variable on which two expressions depend, these are associated with points on the line that are always symmetrical to point 0

The expression indicates a value of 0 for any value that the variable assumes on the line

When a variable is dragged along the line and an associated expression takes on the value 1 , the other expression also assumes the same value

The expression indicates the value 1 for any value that the variable assumes as it is dragged along the line

When the variable $x$ is dragged along the line, the point associated with $E_{1}(x)$ always appears to the right of that associated with $E_{2}(x)$ 
Expression that is always positive (i.e. $x^{2}+1$ )

Constant expression (i.e. $3(x-1)-3 x+5)$
The point associated with the expression $E_{1}(x)$ always appears on the positive side of the line when dragging the variable $x$

The point associated with the expression does not move when the variable $\mathrm{x}$ is dragged along the line

Each of the above narratives is characterised by a set of specific dynamic and visuospatial characteristics that manifest over time through use of the dragging affordance.

Students to solve a task can exploit these characteristics. Moreover, these characteristics have a strong expressiveness of the proprieties of the algebraic relations involved and can be very useful for students to assign the correct meaning to the terms of the natural language that are used to define such properties and to understand how such properties are incorporated in the symbolic language of algebra (Arzarello, Bazzini \& Chiappini, 2001).

Hence, the Bruner's principles are an important tool to investigate the aspects of the narratives that are crucial for the development of the algebraic thought according to two different but integrated perspective (Bottino \& Chiappini, 1998):

- For the emergence of objectives for the task to be solved. In this perspective, the NEUA carries out the function of ergonomic affordance;

- For the construction of meanings, concepts, principles and values of algebra. In this perspective, the NEUA carries out the function of cultural affordance.

Even if these two functions of the NEUA can be strictly integrated in the algebraic activity, each of them keeps its own specificity in the development of the algebraic thought.

Usefulness and expressiveness of the ergonomic and cultural affordances can be recognised only within an algebraic activity. It is, therefore, necessary to understand how the student can come to recognise them and to internalise the ergonomic and cultural affordances of an artefact by developing an algebraic activity.

\section{The cycle of expansive learning as a tool to evaluate the features of the activity}

Analysis of student's behaviours in the use of the AL has helped us to shed light on the conditions under which students may internalise the ergonomic and cultural aspects that are underlying the use of this artefact (Chiappini, 2012).

The method we adopted in order to accomplish this analysis is based on the model of cycle of expansive learning that Engeström has developed in the framework of the Activity Theory (Engeström, 1987, Engeström \& Sannino, 2010).

The notion of cycle of expansive learning was created by Engeström to model the processes of activity transformation that may lead participants to re-conceptualise the object of the activity. The application of this notion allowed us to observe that the critical aspect involved in the internalisation of ergonomic and cultural affordances concerns the identification and the overcoming of contradictions between what emerge by NEUA and what the students had hypothesised on the grounds of their previous knowledge. The contradictions are the result of some feature of the NEUA that the student experiences as an effective surprise. 'Effective surprise', as Bruner stated, is 'the hallmark of a creative enterprise' (Bruner, 1962). Bruner saw the effective surprise as a moment of experience that alters how you see the world.

Experiments carried out with AlNuSet (Chiappini, Reggiani \& Angelini, 2014) have highlighted that an effective surprise has the capacity to undermine the wrong hypothesis formulated by the student for the solution of the problem or to put in discussion meanings, concepts and principles concerning the algebraic objects. Through the questioning of students' conceptions, contradictions emerge in the algebraic activity. Moreover, the experiments have evidenced that the NEUA also assume an important role in overcoming the contradiction generated by an effective surprise because it can contribute to transform the activity and to re-conceptualise its object.

In the experiments carried out, the transformation of the activity took place through a cycle of expansive learning structured into four phases. During these phases of the activity cycle, the NEUA has contributed to create the conditions for the internalisation of ergonomic and cultural affordances of the artefact, for the re-configuration of the object of the activity (Engeström \& Sannino, 2010) and for the learning.

It is important to note that the expansive learning cycle began when a relatively stable pattern of student's behaviour has been questioned by an effective surprise of the NEUA and it ended when a new form of activity has been consolidated and relatively stable. More in particular, the experiments have highlight that

- The first phase of the expansive learning cycle was characterised by the assignment of an open problem on an important issue of algebra learning concerning an obstacle of an epistemological nature. The problem was designed to exploit some aspects of the NEUA to query the resolving hypothesis put forth by students on the grounds of their knowledge. Typically, a conflict emerges because some aspect of the NEUA appear surprising to the students' consciences. In this phase, the teacher helped students in the process of raising their consciousness of the conflict;

- In the second phase, students were requested to face tasks that broaden problematic areas of the knowledge in question. The tasks of this phase were designed in order to exploit the ergonomic affordance of the AL to allow students to explore the conditions, causes and explicative mechanisms of conflicts (Engeström, 2010) that emerged 
in the solution of the tasks. The practices of this phase were modelled to submit the experience in the AL environment to a common reflection and discussion in the class. These were aimed to recognise a usefulness to the NEUA for the problems to be solved and to reach a shared interpretation of the meaning involved in their solution;

- In the third phase, the AL was used in conjunction with AlNuSet's algebraic manipulator (not illustrated in this work). The narratives that emerged from the use of the two artefacts are compared. The teacher's attention was aimed at facilitating symbolic interpretation of what the students had learned through experience with the NEUA of the AL. In this phase, the teacher encouraged the development of the meta-cognitive processes involved in the comparison of the narratives that emerged with the two artefacts;

- In the fourth phase, students became aware of the fact that the activity system had been transformed through the use of the two artefacts. This changed the state of their knowledge of algebraic activity. This phase was characterised by teacher-guided assessment/discussion of classroom activity (Bartolini Bussi, 1998; Mariotti, 2009). Its purpose was to foster students' full awareness of the developed knowledge through comparison with their perceived knowledge state before the cycle began.

The expansive learning cycle that sprang from these developments and from the transformation phases characterised by the above-mentioned conditions of artefact use allowed students to internalise the ergonomic and cultural affordances of the artefact.

\section{Conclusions}

This work illustrates a methodology for evaluating the effectiveness of the educational potential of a digital artefact for the teaching and learning of algebra as determined equally by (i) the structure of actions and representations available within the artefact (embedded by the designer), (ii) the interpretation and behaviour of who uses it and (iii) the features of the activity in which it is used. The methodology has been experimented through AlNuSet, a system designed for supporting the teaching and learning of algebra by means of modalities of interaction that are of visual, spatial and motor nature.

The methodology uses a framework centred on a three-layer articulation of the notion of affordance elaborated by Turner and Turner that distinguishes amongst perceived affordance, ergonomic affordance and cultural affordance of a digital artefact.

Moreover, the methodology draws on Bruner's universal principles of narrative construction of realities in order to analyse and to assess the NEUA in order to grasp the usefulness of the ergonomic affordances and the expressiveness of the cultural affordances of the artefact, respectively, for the solution of algebraic problems and for the appropriation of meaning, concept, principles of the algebra domain.

Finally, the methodology refers to the model of cycle of expansive learning elaborated by Engeström to analyse the role of the NEUA in the algebraic activity. By means of this model, it is possible to identify the conditions of use of the artefact and the forms of collaboration, which can determine a transformation of the algebraic activity. This transformation leads users to redefine the object of the activity and to internalise the ergonomic and the cultural affordances of the artefact.

The work highlights that through the integration of these three types of analysis, it is possible to evaluate the educational potential of the artefact used in the algebraic activity.

Whilst this study has concentrated on a specific artefact, namely, the AlNuSet system, we believe that the method presented here can also be applied to other software and different knowledge domains. However, further studies are needed in order to validate applicability of the methodology to new artefacts and domains.

\section{References}

Arzarello, F., Bazzini, L., \& Chiappini, G. (2001). A model for analyzing algebraic process of thinking. In R. Sutherland, T. Rojano, \& A. Bell (Eds), Perspectives on school algebra. (pp 61-82). Dordrecht, The Netherlands: Kluwer Academic Publisher.

Bartolini Bussi, M.G. (1998). Verbal interaction in mathematics classroom: a Vygotskian analysis. In H. Steinbring, M.G. Bartolini Bussi \& A. Sierpinska (eds.), Language and communication in mathematics classroom. (pp 65-84). Reston, VA: NCTM.

Bottino, R.M., \& Chiappini, G. (1998). User action and social interaction mediated by direct manipulation interfaces. Education and Information Technology, 3, (3-4), 203-216.

Bruner, J. (1997), La cultura dell'educazione, Milano, Feltrinelli (pp.129-173) traduzione di The Culture of Education, Harvard University Press, 1996.

Bruner, J. (1991), The Narrative Construction of Reality, Critical inquiry, vol. 18 (1), 1-21 
Bruner, J. S. (1962). The conditions of creativity. In H. Gruber, G. Terrell \& M. Wertheimer (Eds.), Contemporary approaches to cognition (pp. 1-30). New York: Atherton.

Chiappini, G. (2014). La mediazione semiotica di diagrammi visuo-spaziali e deittici per l'apprendimento dell'algebra. Sistemi intelligenti, 299-332.

Chiappini, G. (2012). The transformation of ergonomic affordances into cultural affordances: the case of the AlNuSet system. International Journal for Technology in Mathematics Education, 19, 4, 135-140.

Chiappini, G. (2011). The role of technology in developing principles of symbolical algebra, Pytlak M., Rowland T., Swoboda E. (eds), Proceedings of the Seventh Congress of the European Society for Research in Mathematics Education, 429-439, Retrieved from http://www.cerme7.univ.rzeszow.pl/WG/3/CERME7_WG3_Chiappini.pdf

Chiappini, G., \& Reggiani, M., Angelini, V. (2014). La nozione di narrazione nella valutazione del potenziale semiotico di un artefatto, Orientamenti Pedagogici, 61, 1, 127-150

Engeström, Y. (1987). Learning by expanding. Helsinki: Orienta-Konsultit Oy.

Engeström, Y., \& Sannino, A. (2010). Studies of expansive learning: Foundation, findings and future challenges, Educational Research Review, 5, 1-24.

Gibson, J. (1979). The Ecological Approach to Visual Perception. Boston: Houghton Mifflin.

Lagrange, J.B. \&, Chiappini, G. (2007). Integrating the Learning of algebra with technology at the European level: Two examples in the ReMath project. In D.P. Pantazzi, \& G. Philippou (eds), Proceedings of the Fifth Congress of the European Society for Research in Mathematics Education (pp 903-912). Retrieved from http://jb.lagrange.free.fr/site/papers/CERME_Lagrange_Chiappini_def1.pdf

Lagrange, J.B. \& Kynigos, C. (eds) (2014). Representing mathematics with digital media: Working across theoretical and contextual boundaries, Educational Studies in Mathematics, 84, 3

Mariotti, M.A. (2009). Artefacts and signs after a Vygotskian perspective: the role of the teacher, ZDM Mathematics Education, 41, 427-440.

McGrenere, J. \& Ho, W. (2000). Affordances: clarifying and evolving a concept, Proceedings of Graphics Interface 2000 (pp 179-186). Retrieved from http://www.graphicsinterface.org/proceedings/2000/177/

Nielsen, J. (1995). Ten Usability Heuristics for User Interface Design, Retrieved from http://www.nngroup.com/articles/ten-usability-heuristics/

Norman D.A., \& Draper S. (1986). User Centered System Design, Hillsdale JY: Lawrence Erlbaum Associates.

Norman, D.A. (1993). Things that make us smart, MA: Addison-Wesley Pub. Co.

Norman, D. (1988). The Psychology of Everyday Things, New York: Basic Books.

Pedemonte B., \& Chiappini G. (2008). ALNUSET: a system for teaching and learning algebra. International Journal of Continuing Engineering Education and Life-Long Learning, 18, (5-6), 627-639.

Turner, P. (2005). Affordance as context, Journal Interacting with Computers, 17, 6, 787-800

Turner, P. \& Turner, S. (2002). An affordance-based framework for CVE evaluation. In: People and Computers XVII The Proceedings of the Joint HCI-UPA Conference (pp 89-104). London: Springer

Whitefield A., Wilson F., \& Dowell J. (1991). A framework for human factors evaluation. Behaviour \& Information Technology, 10, 1, 65-79. 